\title{
Costs and persistence of alpha- 2 adrenergic agonists versus carbonic anhydrase inhibitors, both associated with prostaglandin analogues, for glaucoma as recorded by The United Kingdom General Practitioner Research Database
}

\author{
Philippe Denis' \\ Antoine Lafuma ${ }^{2}$ \\ Gilles Berdeaux ${ }^{3}$ \\ 'Hôpital Edouard Herriot, Lyon, \\ France; ${ }^{2}$ Cemka, Bourg-la-Reine, \\ France; ${ }^{3}$ Alcon France, Rueil- \\ Malmaison, France
}

\begin{abstract}
The persistence and costs of carbonic anhydrase inhibitors + prostaglandin analogues (CAIs + PGAs) vs alpha-2 adrenergic agonists + prostaglandin analogues (alpha-2 agonists + PGAs) were compared, based on The United Kingdom General Practitioner Research Database. Patients with a diagnosis of ocular hypertension, glaucoma, or treated for this, were selected. Selected patients were prescribed CAIs + PGAs or alpha-2 agonists + PGAs. Treatment failure was defined as a prescription change (adding, removing, or replacing glaucoma treatment, or initiating laser or surgery). Times to treatment failure were compared with a Cox model adjusted by a propensity score. Mean patient age was 69.0 years and $47.6 \%$ were males. Treatment failure at 1 year was experienced by $58.8 \%$ receiving CAIs + PGAs and $66.0 \%$ of patients receiving alpha-2 agonists + PGAs $(p<0.001)$. The hazard ratio for failure was 0.82 $(\mathrm{p}<0.001)$ in favor of CAIs + PGAs after adjusting on age, gender, comorbidities, and duration of follow-up. Adjusted annual costs of glaucoma management did not differ significantly between treatments, $£ 440.63$ with alpha- 2 agonists + PGAs and $£ 413.37$ with CAIs + PGAs CAIs + PGAs therapies appear more persistent than alpha-2 agonist + PGA in everyday clinical practice, at a similar cost.
\end{abstract}

Keywords: glaucoma, alpha-2 adrenergic agonists, carbonic anhydrase inhibitor, prostaglandin, effectiveness economics, costs

\section{Introduction}

Glaucoma in various forms occurs in about $2 \%$ of the population aged more than 40 years (Weih et al 2001). It affects children and young adults far less frequently than the elderly. It is estimated that in England and Wales, alone, more than 500,000 people suffer from glaucoma and more than 70 million people are affected worldwide (Broman and Quigley 2006) while uncorrected refractive errors remained the major cause of visual impairment (Resnikoff et al 2004). In the great majority of cases the condition takes a chronic, slowly developing course (primary open angle glaucoma: POAG). Studies have demonstrated that about half of all POAG cases remain undiagnosed in the Western developed countries. The risk of POAG is about four times greater for Afro-American people than for Caucasians.

POAG usually affects both eyes and initially produces few symptoms. Eventually, untreated patients may become aware of severely restricted visual fields, or even a loss of central vision in the worst eye. Although blindness from glaucoma is uncommon, it is wholly or partially the third reason to register on the blind register for England 
and Wales, and is the leading cause of preventable blindness in the UK (Bunce and Wormald 2006). When treated, the probability of glaucoma-related blindness, after treatment of both eyes, was estimated at $9 \%$ at 20 years after diagnosis (Hattenhauer et al 1998). A significant risk factor for glaucoma blindness is advanced loss of vision when the condition is first detected. Appropriate examinations during routine eye tests are therefore essential to detect glaucoma early and prevent significant sight loss.

The annual economic burden of glaucoma in the UK was estimated at $£ 62$ million in direct medical costs in 1994 (Coyle and Drummond 1995). Total annual UK costs of glaucoma, including direct, indirect and social cost estimated for the Blind Association 'Guide Dog' service reached $£ 27$ billion (Guide Dog for the Blind Association).

Observational data show that frequent medication changes increase the costs of glaucoma treatment (Kobelt et al 1998; Denis et al 2004). Although the daily cost of a drug must be considered when prescribing (Jönsson and Krieglstein 1998; Fiscella et al 1999; Vold et al 2002; Rylander and Vold 2008), the impact of efficacy should not be overlooked as this can diminish the need for other medical resources, such as ophthalmologist visits, surgery, or laser therapy (Kooner and Zimmerman 1987).

Increased intraocular pressure (IOP) is the main risk factor whereas the other causes of glaucomatous optic neuropathy remain unknown. Various strategies in current use decrease IOP and so lower the risk of glaucoma, thereby limiting its development (Kass et al 2002) and controlling progression (Heijl et al 2002; Leske et al 2003; The AGIS investigators 2004; EGPS 2005). Surgery, laser therapy and several classes of pharmacological agents in common use have been introduced in recent decades. Medications include beta-blockers (marketed in the late 1970s), carbonic anhydrase inhibitors (CIAs) in the mid-1990s, and both alpha-2 adrenergic agonists and prostaglandin analogues (PGAs) in the late 1990s. It is an accepted practice to start treatment with a single drug, followed by drug combinations (whether, or not, fixed) after an initial failure. A second-line monotherapy may be also used in case of failure of the first line monotherapy. Randomized clinical trials of brimonidine vs brinzolamide both associated with travoprost showed a better IOP control in the patients treated with travoprost and brinzolamide (Reis et al 2006; Feldman et al 2007).

Health authorities increasingly demand documentation showing drug efficacy based on prescriptions in everyday practice (Hjelmgren et al 2001). Retrospective cohort studies comparing treatments can be effective strategies to provide such information. Studies using the endpoint of 'persistence' or 'adherence' to treatment are now being applied to glaucoma treatment (Deschaseaux-Voinet et al 2003; Nordstrom et al 2005; Covert and Robin 2006; Wilensky et al 2006).

An extensive source of patient data is The United Kingdom General Practitioner Research Database (UK-GPRD) which collects information from a representative sample of general practitioners (GPs) (http://www.gprd.com/home/). Glaucoma data, for example, are collected comprehensively because GPs perform a gatekeeper role in the UK. This paper compares the costs and effectiveness of two medications (alpha-2 adrenergic agonists vs carbonic anhydrase inhibitors) each used in combination with a prostaglandin analogue as glaucoma therapy.

\section{Materials and methods \\ Data sources}

This study extracted data from the UK-GPRD, which is the world's largest computerized database with anonymous longitudinal records of approximately three million patients in primary medical care collected continuously by general practitioners since 1987. Records include demographic information (other than race) on age, gender, medical diagnosis, prescriptions, clinical events, and hospital referrals.

\section{Data management}

The UK-GPRD mainly reports incidences (eg, visits, drugs, comorbidities), hence considerable data management must be performed to extrapolate the prevalence of events.

Treatments are, here, represented by product names and dates of prescription. The main assumption was that carbonic anhydrase inhibitors (CAIs) or alpha-2 agonists were prescribed for a maximum period of 100 days which approximately corresponds to three eye-drop bottles. Initial prescriptions rarely exceeded this quantity. Two consecutive prescriptions for less than 100 days each were presumed to represent renewed therapy. Any other ophthalmic product prescribed during the same period was regarded as an additional treatment.

\section{Patient selection and inclusion}

Three sets of terms were used to identify the UK-GPRD population of patients treated for glaucoma or intra-ocular hypertension, as follows: (1) Diagnosis required specific 'open-angle' and more generic 'glaucoma' terms that excluded specifically 'closed-angle', 'angle closure' and 'glaucoma due to other causes' (eg, trauma); (2) Surgery or laser terms for glaucoma were flagged as specific 
(eg, 'laser trabeculoplasty' and 'iridectomy for glaucoma') or non-specific (eg, 'iridectomy' not stated as for glaucoma); (3) Medication terms included topical or systemic glaucoma products defined by their active constituents as listed in the British National Formulary.

Patients of either sex were identified by at least one GP visit documenting a clinical referral or test related to a glaucoma diagnostic, surgical or laser term, as above, or a prescription for a specific glaucoma product. Patients with all the following characteristics were included in the analysis: (1) a glaucoma diagnosis (as above), (2) initial treatment with either a CAI + PGA or an alpha-2 agonist + PGA, and (3) follow-up longer than 6 months once the study therapies were begun. Patients with pre-existing CAI or alpha-2 agonist treatments were excluded.

\section{Evaluation criteria}

Treatment failure was defined as a prescription change replacing or discontinuing the initial medication, or adding a new medication (association). Patients given laser therapy or surgery for glaucoma were regarded as treatment failures.

\section{Statistical methods}

Statistical analyses used SAS V9.1 software (SAS Institute; North Carolina, USA). Treatment groups were compared at baseline on demography, general comorbidities and eye comorbidities. Continuous variables were compared by a t-test after verifying normality or by a non-parametric Wilcoxon test, in the absence of normality. Discontinuous variables were analyzed by a Chi-square test that was replaced by Fisher's exact permutation test when samples were small. All tests were interpreted two-sided with $\alpha=0.05$.

Survival curves by treatment group were estimated using the Kaplan-Meier method, without adjustment. The treatment failure hazard-ratio between alpha-2 agonist and CAI was estimated by a Cox regression, with and without adjustment.

Unbalanced confounding factors of treatment efficacy were adjusted for, using the propensity score method (Yue 2007). The propensity score, defined as the conditional probability of being treated with a specific treatment according to the observed covariates, was used to balance covariates in the two treatment groups and so control bias. A logistic regression was performed to estimate a propensity score (probability to be treated with either alpha-2 agonist or CAI) for each patient. Variables (demographics, general and eye co-morbidity, time since diagnosis) included in the logistic regression were selected from univariate statistics searching for treatment unbalances. Then, a stepwise algorithm was used to select variables with an entry and exit p-value fixed at 0.10 . Quartiles based on the estimated propensity score were created and included in the Cox model to obtain an adjusted estimate of the treatment effect (time to failure).

Collected medical resource utilization was specific to glaucoma and therefore should not be impacted by comorbidities, by definition. The only known factors contributing to cost is patient follow-up duration. Two sets of estimates were obtained by adjusting, or not adjusting, on the duration of follow-up. Adjustment was made by a linear regression model: (1) the simple model only included treatment group (2) the adjusted model included treatment group and unbalanced confounding factors.

\section{Economic evaluation}

Direct and consequential costs of glaucoma medical care were analyzed from the economic perspective of the UK National Health Service.

The UK-GPRD yielded the following glaucoma information: (1) laser therapy and surgery, (2) hospitalizations for glaucoma, (3) glaucoma medication, (4) visits to GPs and glaucoma specialists, and (5) prescription renewals by telephone. Consumption items were collected for each patient from the prescription to the final follow-up day and included treatment subsequent to medication failure.

Unit costs of medication were taken from the British National Formulary (2005) (www.bnf.com) and did not include drug discounts. Unit costs for medical procedures (surgery, laser therapy, hospitalization, and consultations) were taken from Jönsson and Krieglstein (1998) and inflated to the year 2005 by applying the National Health Service inflation rate (Statistics of OECD). Visits to ophthalmologists were costed at $£ 107$ per patient hour and follow-up visits were assumed to last 15 minutes (Curtis and Netten A 2005). General practitioner visits were costed at $£ 129$ per patient hour and follow-up visits were assumed to last 15 minutes (Curtis and Netten 2005). We estimated both the total and annual costs of each treatment regimen.

\section{Results}

In total, 54,513 glaucoma patients were selected from the UK-GPRD representing all UK regions. A subgroup of 6,176 patients met our study criteria, comprising 4,792 treated with CAIs + PGAs and 1,384 with alpha-2 agonists + PGAs. The $3: 1$ of ratio CAIs/alpha- 2 agonists showed that CAIs + PGAs were prescribed more frequently for glaucoma than alpha-2 agonists + PGAs. 
No statistically significant differences in gender distribution (47.6\%) or age (mean 69.0 years) were found at inclusion between patients treated with CAIs or alpha-2 agonists (Table 1). However, time since diagnosis was significantly $(\mathrm{p}<0.001)$ longer for the CAI group, whereas the mean duration of follow-up was significantly $(\mathrm{p}<0.001)$ longer for alpha-2 agonist group (1,060 days) than the CAI group (838 days).

General health and eye comorbidity data, comprising all prevalent and incident events from entry into the UKGPRD to the exit date of the present study, are presented in Table 2. Statistically significant differences were noted between the two treatment groups. As compared to CAIs, patients treated with alpha-2 agonists experienced more ocular sensory symptoms and general comorbidities, ie, cardiac disorders, immune system disorders, nervous system disorders, psychiatric disorders, and respiratory disorders. Neither category of comorbidities was associated with time to treatment failure.

One year after starting glaucoma medication, patients experienced less treatment failure with CAIs than with alpha-2 agonists (Table 3) and this advantage was maintained throughout the subsequent 3 years. Accordingly, the hazard ratio for failure was significantly less with CAIs $(\mathrm{OR}=0.82: \mathrm{p}<0.0001)$ before adjustment and remained so after adjustment for age, gender, comorbidities, and duration of follow-up (OR $=0.82: \mathrm{p}<0.001)$. Time to $50 \%$ failure was longer with CAIs ( 8.5 months). Survival curves are shown in Figure 1.

Most of the patients were followed up until failure of the current treatment-line: both treatments failed for 5,734 of 6,176 patients ( $92.8 \%$ ) during the study period (Table 4 ). Second-line treatment for more than half of all patients was a switch to prostaglandin analogues (in monotherapy or in combination, with beta-blockers the next preference) and for $2.9 \%$ a switch to surgery or laser therapy.

Average monthly costs associated with CAIs + PGAs were estimated at $£ 33.98$ per patient and were close to those of alpha-2 agonists + PGAs, $£ 36.22$ (Table 5). There was no significant difference between these costs even after adjustment. Average daily costs with CAIs were $98.2 \%$ of those with alpha-2 agonists. The adjusted annual cost difference of a patient receiving CAI treatment was only $£ 27.26$ less than for an alpha-2 agonist.

\section{Discussion}

The use of carbonic anhydrase inhibitors and prostaglandin analogues was compared in this study with alpha-2
Table I Patient demography and follow-up duration

\begin{tabular}{|c|c|c|c|}
\hline Variable & $\begin{array}{l}\text { Alpha-2 agonists } \\
\mathrm{n}=\mathrm{I}, 384\end{array}$ & $\begin{array}{l}\text { CAls } \\
n=4,792\end{array}$ & p-value \\
\hline \multicolumn{4}{|l|}{ Gender } \\
\hline Male & $47.8 \%$ & $47.5 \%$ & 0.8466 \\
\hline Female & $52.2 \%$ & $52.5 \%$ & \\
\hline Age (years) & $69.2(12.0)$ & $68.9(12.5)$ & 0.6419 \\
\hline $\begin{array}{l}\text { Time since diagnosis } \\
\text { (years) }\end{array}$ & $4.08[2.94]$ & 4.51 [3.47] & $<0.001$ \\
\hline $\begin{array}{l}\text { Mean duration of } \\
\text { follow-up (days) }\end{array}$ & I,059.6 [967] & $838.1[700]$ & $<0.001$ \\
\hline
\end{tabular}

Note: (sd) [median]

Abbreviation: CAls, carbonic anhydrase inhibitors.

adrenergic agonists and prostaglandin analogues during everyday UK general practice.

Many researchers claim that non-randomized studies lead to unreliable results and advocate the unique use of randomized controlled trials (RCTs) (Dunn et al 2002). Despite the risk of producing biased treatment estimates, other authors favor the use of non-randomized studies on the basis that these can lead to results which may be compared with those reported in RCTs (Concato et al 2000). Therefore, sources of existing observational data can provide valuable information. Also, RCTs are generally conducted under strict protocol conditions that differ from everyday practice. Consequently, some authors assert that randomized studies do not provide information of much relevance to decisionmakers (Heckman et al 1995). Thus, while most clinical researchers regard observational studies as exploratory tools yielding results that need to be confirmed by RCTs, non-randomized studies need to be performed after RCTs to assess the external validity of RCT findings. Our database analysis should be interpreted in this manner.

On the information available, we observed no sociodemographic differences between patients receiving CAIs or alpha-2 agonists, except for time since diagnosis and duration of follow-up, despite the lack of treatment randomization. The latter is explained by the earlier Market Access Authorization of brimonidine (European Medicines Agency). General and ocular comorbidity differences between the treatment groups were not related to clinical outcomes and no adjustment was necessary. Nonetheless, we analyzed treatment failure both with and without adjustment and obtained very similar results, confirming the robustness of our findings. In addition, we used a propensity score analysis which is well accepted by health authorities (Yue 2004). This approach follows the recommendations issued by the British Medical Journal (Mamdani et al 2005; Normand et al 2005; Rochon et al 2005). 
Table 2 Eye and general comorbidities - UK GPRD prevalence rates

\begin{tabular}{|c|c|c|c|}
\hline Comorbity & Alpha-2 agonists & CAls & p-value \\
\hline \multicolumn{4}{|l|}{ Ocular comorbidity } \\
\hline Anterior eye structural changes & $273(19.7 \%)$ & $\mathrm{I}, 0 \mathrm{I} 5(2 \mathrm{I} .2 \%)$ & 0.2403 \\
\hline Congenital eye disorders & $10(0.7 \%)$ & $36(0.8 \%)$ & 0.9129 \\
\hline Ocular infections, irritations and inflammations & $444(32.1 \%)$ & $1,459(30.4 \%)$ & 0.2461 \\
\hline Ocular injuries & $20(1.4 \%)$ & $66(1.4 \%)$ & 0.8496 \\
\hline Ocular neuro-muscular disorders & $14(1.0 \%)$ & $55(1.1 \%)$ & 0.6711 \\
\hline Ocular sensory symptoms & $12(0.9 \%)$ & $15(0.3 \%)$ & 0.0059 \\
\hline Retina, choroid and vitreous hemorrhages and vascular disorders & $101(7.3 \%)$ & $319(6.7 \%)$ & 0.4043 \\
\hline Ocular hemorrhages and vascular disorders & $64(4.6 \%)$ & 177 (3.7\%) & 0.1153 \\
\hline Vision disorders & $262(18.9 \%)$ & 884 (I8.4\%) & 0.6838 \\
\hline \multicolumn{4}{|l|}{ General comorbidity } \\
\hline Blood and lymphatic disorders & $162(11.7 \%)$ & $618(12.9 \%)$ & 0.2399 \\
\hline Cardiac disorders & $698(50.4 \%)$ & $2,184(45.6 \%)$ & 0.0014 \\
\hline Congenital and genetic disorders & $73(5.3 \%)$ & $279(5.8 \%)$ & 0.4389 \\
\hline Ear disorders & $645(46.6 \%)$ & $2,224(46.4 \%)$ & 0.8989 \\
\hline Endocrine disorders & $289(20.7 \%)$ & $978(20.4 \%)$ & 0.7900 \\
\hline Gastrointestinal & $912(65.9 \%)$ & $3,182(66.4 \%)$ & 0.7256 \\
\hline Liver disorder & $69(5.0 \%)$ & $228(4.8 \%)$ & 0.7274 \\
\hline Immune system disorders & $700(50.6 \%)$ & $2,182(45.5 \%)$ & $<0.001$ \\
\hline Infections & $1,036(74.9 \%)$ & 3,524 (73.5\%) & 0.3264 \\
\hline Injuries & $630(45.5 \%)$ & $2,144(44.7 \%)$ & 0.6078 \\
\hline Metabolism and nutrition disorders & $633(45.7 \%)$ & $2,212(46.2 \%)$ & 0.7808 \\
\hline Musculo-skeletal disorders & I,083 (78.3\%) & $3,722(77.7 \%)$ & 0.6472 \\
\hline Neoplasm benign and malignant & $477(34.5 \%)$ & $\mathrm{I}, 709$ (35.7\%) & 0.4116 \\
\hline Nervous system disorders & $892(64.5 \%)$ & $2,898(60.5 \%)$ & 0.0075 \\
\hline Psychiatric disorders & $547(39.5 \%)$ & $2,038(42,5 \%)$ & 0.0458 \\
\hline Renal and urinary disorders & $543(39.2 \%)$ & $1,879(39.2 \%)$ & 0.9877 \\
\hline Respiratory disorders & $1,079(78.0 \%)$ & $3,570(74.5 \%)$ & 0.0085 \\
\hline Skin and subcutaneous tissue disorders & $932(67.3 \%)$ & $3,212(67.0 \%)$ & 0.8274 \\
\hline
\end{tabular}

Abbreviation: CAls, carbonic anhydrase inhibitors.

As far as possible, taking into account the information available in the UK GPRD database, we gave due attention to controlling selection bias and confounding factors. First, these matters are clinically relevant as both drug classes had the same indications. Moreover, the choice between a CAI, or an alpha-2 agonist combined with a PGA, after the latter monotherapy has failed, is clinically important. Second, data were collected on available factors known to be confounded with treatment persistence, eg, age as a surrogate endpoint of glaucoma severity, eye, and general co-morbidities. Data on other confounding factors were not recorded in the database (eg, race and IOP at treatment initiation). However, as the estimated propensity score may be regarded as a latent variable, summarizing disease severity, the reported findings were duly adjusted. Third, baseline comparison was performed and showed no major differences between the treatment groups. Fourth, none of the few unbalanced variables was linked to treatment persistence. Hence, our propensity score might have led to some over-adjustment, despite which the adjusted results remained statistically significant.
Lastly, two adjustment procedures, ie, regression (not reported) and propensity scores, were used and neither showed any major change of treatment effect.

The larger number of patients treated with a CAI could have introduced a channelling bias, ie,the few severe cases would have been treated with the most effective drug. We believe that two major factors would explain this imbalance: (1) two CAIs were marketed (brinzolamide and dorzolamide), as compared to one of the alpha- 2 agonists (brimonidine), and (2) dorzolamide market authorization and access preceded brimonidine by 1 year.

The number of drugs following CAI or alpha-2 agonist treatment failure was somewhat greater after the alpha-2 agonists, suggesting a more frequent occurrence of severe cases in the latter patient group. From a strict standpoint, treatment group imbalance should be considered at the initiation of treatments. Therefore, we did not regard subsequent events as factors influencing treatment persistence. Also, the initiation of a rescue treatment depends on the previous treatment and the choice should take into account the 
Table 3 Adjusted failure rates over time according to treatment

\begin{tabular}{lll}
\hline Duration of treatment & Alpha-2 agonists & CAls \\
\hline 12 months & $34.0 \%$ & $41.2 \%$ \\
24 months & $16.4 \%$ & $22.6 \%$ \\
36 months & $8.1 \%$ & $12.7 \%$ \\
48 months & $3.5 \%$ & $6.3 \%$ \\
Time to 50\% failure incidence & 6.5 & 8.5 \\
rate (month) & & \\
\hline
\end{tabular}

Abbreviation: CAls, carbonic anhydrase inhibitors.

pharmaco-dynamics of the drugs concerned, which differ between CAIs and alpha-2 agonists. Lastly, the number of patients given surgery or laser therapy as rescue treatments, ie, the most severe cases, was similar.

Data management was performed 'blind', before any patient was selected, with respect to the general glaucoma database where all types of treatment were pooled together. This allowed us to minimize bias when comparing treatments. Also, this approach was meaningful as the compared glaucoma drugs were homogenously distributed, hence choices made at a global level should not have impacted on any specific treatment. We performed sensitivity analyses on subsets of the entire glaucoma database (eg, when selecting the 100-day threshold) because our data management programs required intensive computer resources (some files exceeded 15 millions lines). Accordingly, we believe that our data management was fair and did not systematically bias treatment comparisons.

Treatment failure was signaled by a change of treatment, ie, adding, stopping or replacing glaucoma medication, surgery or laser therapy, as is common practice with glaucoma analyses of claim data (Deschaseaux-Voinet et al 2003; Nordstrom et al 2005; Covert and Robin 2006; Wilensky et al 2006).

We found a significantly lower rate of treatment failure with CAIs + PGAs over a 4 year period, according to our outcome definition. The comparison between treatment persistence and clinical efficacy is not straightforward since the former encompasses several dimensions which are usually controlled in clinical trials (eg, treatment delivery, compliance, efficacy, safety). Our results could be compared to those of Feldman et al (2007) and Reis et al (2006), who reported randomized, masked studies, comparing dorzolamide and brimonidine. Both sets of authors concluded that the treatments produced equivalent IOP control. Our different observation may be explained by several factors. First, we studied treatments in contrast to the two randomized trials, which concerned second-line treatments. Second, our study

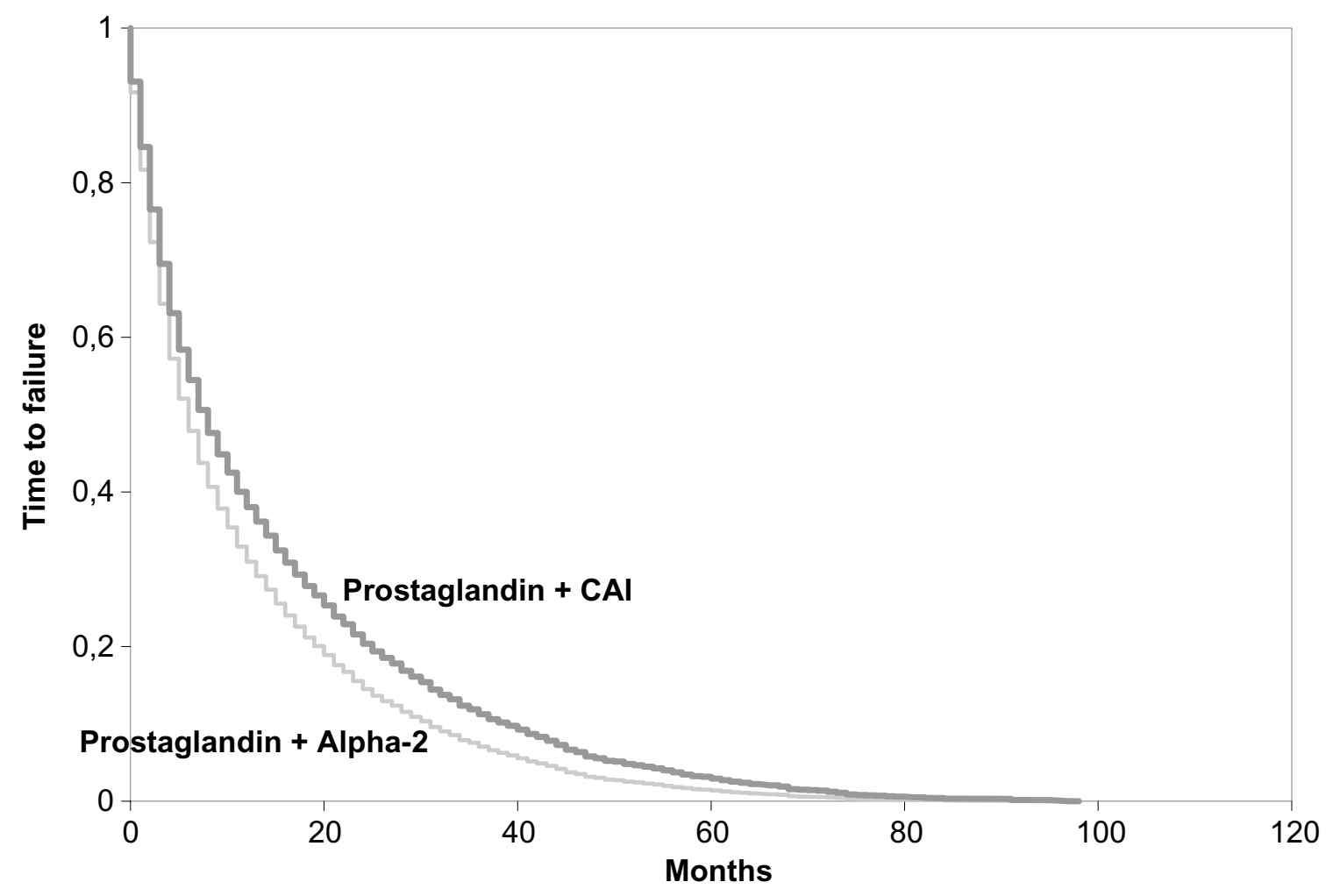

Figure I Time to failure of carbonic anhydrase inhibitors (CAls) and alpha-2 agonists from the initiation of therapy. 
Table 4 Resource utilization after $\mathrm{CAl}$ alpha-2 agonist treatment failure

\begin{tabular}{|c|c|c|c|}
\hline $\begin{array}{l}\text { Rescue } \\
\text { treatment }\end{array}$ & $\begin{array}{l}\text { Alpha-2 agonists } \\
n=1,319\end{array}$ & $\begin{array}{l}\text { CAls } \\
n=4,415\end{array}$ & $\begin{array}{l}\text { Total } \\
n=5,734\end{array}$ \\
\hline \multicolumn{4}{|l|}{ Number of drugs } \\
\hline No drugs & $39(3.0 \%)$ & 126 (2.9\%) & 165 (2.9\%) \\
\hline Monotherapy & $854(64.7 \%)$ & $3,280(74.3 \%)$ & $4,134(72.1 \%)$ \\
\hline 2 drugs & 197 (14.9\%) & 435 (9.9\%) & $632(11.0 \%)$ \\
\hline 3 drugs & $223(16.9 \%)$ & $553(12.5 \%)$ & $776(13.5 \%)$ \\
\hline 4 drugs & $6(0.5 \%)$ & $21(0.5 \%)$ & $27(0.5 \%)$ \\
\hline Surgery & $32(2.4 \%)$ & $110(2.5 \%)$ & $142(2.5 \%)$ \\
\hline Laser therapy & $7(0.5 \%)$ & $16(0.3 \%)$ & $23(0.4 \%)$ \\
\hline \multicolumn{4}{|l|}{ Drug classes } \\
\hline Beta-blocker & 270 (20.5\%) & I,529 (34.6\%) & I,799 (31.4\%) \\
\hline $\begin{array}{l}\text { Prostaglandin } \\
\text { analogue }\end{array}$ & $845(64.1 \%)$ & 2,732 (6I.9\%) & 3,577 (62.4\%) \\
\hline Alpha-2 & $663(50.3 \%)$ & 345 (7.8\%) & I,008 (I7.6\%) \\
\hline CAI & 249 (18.9\%) & 227 (5.1\%) & $476(8.3 \%)$ \\
\hline
\end{tabular}

Abbreviation: CAls, carbonic anhydrase inhibitors.

involved large patient populations recorded in the UK-GPRD which provided greater power to observe small significant differences. Third, treatment acceptability may play a role. Although Stewart et al found both treatments to be well tolerated and without significant effects on safety parameters (Stewart et al 2000), Whitson et al (2004) observed significantly more stinging and burning with dorzolamide (CAI) $(\mathrm{p}<0.02)$ and more frequent dry eyes with brimonidine (alpha-2 agonist) ( $\mathrm{p}=0.04$ ) (Whiston et al 2004). Accordingly, in everyday practice it is possible that treatment failure may be related to unwanted effects that patients will not tolerate. Specific patient chart data should be collected to test this hypothesis.

The costs of CAIs + PGA were higher than the ones of alpha-2 + PGA while the cost of one bottle of brimonidine was higher (about $+20 \%$ ) than the average cost of the two
CAIs participating in the analysis. This was explained by the longer treatment persistence of CAIs. Treatment failure leads to higher costs (Denis et al 2004) as confirmed, here, by the nature of the rescue treatment which in most cases comprised the more costly prostaglandin analogues. Conversely, medications that would lower the failure rate should reduce the costs of patients who receive them. The annual failure rate with CAIs was significantly lower over 4 years than for alpha-2 agonists. Hence, by delaying the need for more expensive glaucoma treatments, CAIs would eventually become less expensive than alpha-2 agonists. Results for adjusted and non-adjusted costs were presented because the follow-up time for patients given CAIs was longer than with alpha-2 agonists. Cost differences during either drug regimen remained non-significant both with and without adjustment.

Our analysis has the following limitations. First, as prescription renewal was not clearly documented we fixed the average renewal period at 100 days, corresponding approximately to three eye-drop bottles. An eye-drop bottle contains fluid for 28 days according to the summary of product characteristics (Vidal 2006), with extra fluid to replace for poor instillations by elderly persons (Roulland et al 2003). Thus, our assumption of 100 days per prescription appears reasonable. In order to confirm our findings, experimental designs with prospective data collection are required to document in detail treatment switches and the reasons for switches. Hence, observational surveys recording treatment administration prospectively might be of scientific interest.

Second, reasons for modifying treatments are not documented in the UK-GPRD, and that limits a definitive understanding. Given this circumstance, it was assumed that treatment changes were made for reasons of tolerance or lack of efficacy.

Table 5 Annual average cost of glaucoma according to the treatment ( $(: 2005)$

\begin{tabular}{|c|c|c|c|}
\hline Cost item & Alpha-2 agonists & CAls & p-value \\
\hline Annual total costs from inclusion & $413.83(700.37)$ & $421.11(762.14)$ & 0.7501 \\
\hline Monthly total costs from inclusion & 34.01 (57.56) & $34.61(62.64)$ & 0.7501 \\
\hline Drugs & $23.83(36.91)$ & $24.44(36.93)$ & $\sim 1$ \\
\hline Monthly costs of drugs of study & $7.29(15.21)$ & $9.19(14.55)$ & $<0.0001$ \\
\hline Monthly costs of other drugs & $16.54(22.36)$ & $15.25(23.24)$ & 0.0657 \\
\hline Monthly costs of consultation & $8.01(19.45)$ & $8.30(20.11)$ & 0.6242 \\
\hline Monthly costs of hospitalization-surgery-laser therapy & $2.17(16.12)$ & $1.88(24.66)$ & $\sim 1$ \\
\hline Mean daily costs unadjusted & $1.13(1.92)$ & $1.15(2.09)$ & 0.7501 \\
\hline \multirow[t]{2}{*}{ Annual adjusted total costs } & 440.63 & 413.37 & 0.2327 \\
\hline & [40 $1.27-480.0]$ & [392.32-434.4I] & \\
\hline
\end{tabular}

Note: (sd) [Cl 95\%]

Abbreviation: CAls, carbonic anhydrase inhibitors. 
Third, the main limitation of this study was its retrospective design, which precluded any verification of data accuracy. Conversely, we were able to exploit aspects of UK National Health Service regulations that restrict a patient's access to other physicians, provide longitudinal data, and ensure a high standard of documentation which does not always apply to other health organizations. Hence, we were able to document previous treatments and check group comparability accurately, even though the internal validity of our survey was not equal to that of a randomized clinical trial.

\section{Conclusion}

According to the UK-GPRD information, glaucoma treatment with CAIs + PGAs was more persistent in everyday practice than alpha-2 agonists + PGAs. Patients continued longer with CAIs. During 4 years of treatment the daily costs of a patient receiving CAIs were similar to those of alpha-2 agonists.

\section{Acknowledgments}

This analysis was supported by an unrestricted grant from Alcon France SA, Rueil-Malmaison, France. The analysis was performed by Cemka Eval, Bourg-la-Reine, France. Dr Gilles Berdeaux is employed by Alcon France. We would like to thank Robert Pigache MD, FFPM for reviewing the English language.

\section{References}

Broman AT, Quigley HA. 2006. The number of people with glaucoma worldwide in 2010 and 2020. Br J Ophthalmol, 90:253-4.

Bunce C, Wormald R. 2006. Leading causes of certification for blindness and partial sight in England and Wales. BMC Public Health, 6:58.

Concato J, Shah N, Horwitz RI. 2000. Randomized, controlled trials, observational studies, and the hierarchy of research designs. $N$ Engl J Med, 342:1887-92.

Covert D, Robin AL. 2006. Adjunctive glaucoma therapy use associated with travoprost, bimatoprost, and latanoprost. Curr Med Res Opin, 22:971-6.

Coyle D, Drummond M. 1995. The economic burden of glaucoma in the UK. The need for a far-sighted policy. Pharmacoeconomics, 7:484-9.

Curtis L, Netten A. Units costs of health and social care 2005 PSSRU University of Kent. (http://www.pssru.ac.uk/)

Denis P, Lafuma A, Berdeaux G. 2004. Medical predictive factors of glaucoma treatment costs. J Glaucoma, 13:283-90.

Deschaseaux-Voinet C, Lafuma A, Berdeaux G. 2003. Cost and effectiveness of brinzolamide versus dorzolamide in current practice: an analysis based on the UK-GPRD data base. J Med Econom, 6:69-78.

Dunn D, Babiker A, Hooker M, Darbyshire J. 2002. The dangers of inferring treatment effects from observational data: a case study in HIV infection. Control Clin Trials, 23:106-10.

European Medicines Agency. European Public Assessment Report (EPAR). http:/www.emea.europa.eu/htms/human/epar/a.htm

Feldman RM, Tanna AP, Gross RL, et al; Additivity Study Group. 2007. Comparison of the ocular hypotensive efficacy of adjunctive brimonidine $0.15 \%$ or brinzolamide $1 \%$ in combination with travoprost $0.004 \%$. Ophthalmology, 114:1248-54.
Fiscella RG, Geller JL, Gryz LL, et al. 1999. Cost considerations of medical therapy for glaucoma. Am J Ophthalmol, 128:426-33.

Heckman JJ, Smith JA. 1995. Assessing the case for social experiments. $J$ Econ Perspect, 9:85-110.

Heijl A, Leske MC, Bengtsson B, et al. 2002. Early Manifest Glaucoma Trial Group. Reduction of intraocular pressure and glaucoma progression: results from the Early Manifest Glaucoma Trial. Arch Ophthalmol, 120:1268-79.

Hjelmgren J, Berggren F, Andersson D. 2001. Health economics guidelines - Similarities, differences and some implications. Health Values, 4:225-50.

Jönsson B, Krieglstein G. 1998. Primary open-angle glaucoma-differences in international treatment patterns and costs. Oxford: Isis Medical Media Ltd.

Hattenhauer MG, Johnson DH, Ing HH, et al. 1998. The probability of blindness from open-angle glaucoma. Ophthalmology, 105:2099-104.

Kass MA, Heuer DK, Higginbotham EJ, et al. 2002. The Ocular Hypertension Treatment Study: A randomized trial determines that topical ocular hypotensive medication delays or prevents the onset of primary open-angle glaucoma. Arch Ophthalmol, 120:701-13.

Kobelt G, Jonsson L, Gerdtham U, et al. 1998. Direct costs of glaucoma management following initiation of medical therapy. A simulation model based on an observational study of glaucoma treatment in Germany. Graefes Arch Clin Exp Ophthalmol, 236:811-21.

Kooner KS, Zimmerman TJ. 1987. The cost of antiglaucoma medications. Ann Ophthalmol, 19:327-8.

Leske MC, Heijl A, Hussein M, et al. 2003. Early Manifest Glaucoma Trial Group Factors for glaucoma progression and the effect of treatment: the early manifest glaucoma trial. Arch Ophthalmol, 121:48-56.

Mamdani M, Sykora K, Li P, et al. 2005. Reader's guide to critical appraisal of cohort studies: 2. Assessing potential for confounding. BMJ, 330:960-2.

Nordstrom BL, Friedman DS, Mozaffari E, et al. 2005. Persistence and adherence with topical glaucoma therapy. Am J Ophthalmol, 140:598-606.

Normand SL, Sykora K, Li P, et al. 2005. Readers guide to critical appraisal of cohort studies: 3 . Analytical strategies to reduce confounding. $B M J$, 330:1021-3.

Reis R, Queiroz CF, Santos LC, et al. 2006. A randomized, investigatormasked, 4-week study comparing timolol maleate $0.5 \%$, brinzolamide $1 \%$, and brimonidine tartrate $0.2 \%$ as adjunctive therapies to travoprost $0.004 \%$ in adults with primary open-angle glaucoma or ocular hypertension. Clin Ther, 28:552-9.

Resnikoff S, Pascolini D, Mariotti SP, et al. 2008. Global magnitude of visual impairment caused by uncorrected refractive errors in 2004. Bull WHO, 86:63-70.

Rochon P, Gurwitz J, Sykora K, et al. 2005. Reader's guide to critical appraisal of cohort studies: 1. Role and design. BMJ, 330:895-7.

Roulland JF, Le Pen C, Gouveia Pinto, et al. 2003. Cost-minimisation study of dorzolamide versus brinzolamide in the treatment of ocular hypertension and primary open glaucoma in 4 European countries. Pharmacoeconomics, 21:201-13.

Rylander NR, Vold SD. 2008. Cost analysis of glaucoma medications. Am J Ophthalmol, 145:106-13.

Statistics of OECD. URL: http://www.oecd.org/

Stewart WC, Sharpe ED, Harbin TS et al. 2000. Brimonidine 0.2\% versus dorzolamide $2 \%$ each given three times daily to reduce intraocular pressure. Am J Ophtalmol, 129:723-7.

The AGIS investigators. 2004. The Advanced Glaucoma Intervention Study (AGIS). Report \#13: Comparison of treatment outcomes within race: 10-year results. Ophthalmology, 111:651-64.

The Guide Dog for the Blind Association. The costs of blindness: an analysis of the costs of visual impairment and blindness in the United Kingdom. Accessed April 2004. URL: http://www.healthyeyes.org. uk/file admin/downloads/cost-of-blindness.doc.

[EGPS] The European Glaucoma Prevention Study (EGPS) group. 2005. Results of the European Glaucoma Prevention Study. Ophthalmology, 112:366-75. 
Vidal. 2006. Edition du Vidal. Paris, France.

Vold SD, Riggs WL, Jackimiec J. 2002. Cost analysis of glaucoma medications: a 3-year review. J Glaucoma, 11:354-8.

Weih LM, Nanjan M, McCarty CA, et al. 2001. Prevalence and predictors of open-angle glaucoma: results from the visual impairment project. Ophthalmology, 108:1966-72.

Whitson JT, Henry C, Hughes B et al. 2004. Comparison of the safety and efficacy of dorzolamide $2 \%$ and brimonidine $0.2 \%$ in patients with glaucoma or ocular hypertension. J Glaucoma, 13:168-73.
Wilensky J, Fiscella RG, Carlson AM, et al. 2006. Measurement of persistence and adherence to regimens of IOP-lowering glaucoma medications using pharmacy claims data. Am J Ophthalmol, 141(1 Suppl):S28-33.

Yue LQ. 2007. Statistical and regulatory issues with the application of propensity score analysis to nonrandomized medical device clinical studies. J Biopharm Stat, 17:1-13; discussion 15-7, 19-21, 23-7 passim. 
